\title{
Inferior Rectus Muscle
}

National Cancer Institute

\section{Source}

National Cancer Institute. Inferior Rectus Muscle. NCI Thesaurus. Code C32790.

One of the extraoculomotor muscles, which arises from a common tendinous ring in the posterior portion of the orbit, inserts on the anterior globe, is innervated by CN III, and functions to depress and adduct the eye. 